\title{
Neurocomputing research in The Netherlands
}

\author{
H. Kappen and C. Gielen \\ Department of Medical Physics and Biophysics, University of Nijmegen, Geert Grooteplein Noord 2I, NL 652.5 EZ \\ Nijmegen, Netherlands
}

\section{Introduction}

Neural networks have come a long way. 50 years after the original work by McCulloch, Pitts, Hebb, and von Neumann, the idea of a large network of simple "neuron-like" processors as a computational and dynamical model for the brain, and indeed, for parallel processing in general, is still very alive. In these last fifty years neural networks has become a truly multi-disciplinary field of research with applications in various areas.

In cognition and experimental psychology, neural networks have been succesfully applied to model some typical human performances, such as associative memory, speech production and speech perception, and visual pattern recognition. Several of these neural networks have been implemented in hardware.

In biophysics and physiology, neural networks are built as models of the neural structures that are observed in parts of the brain. It has been shown, that some of the observed anatomical connections in biological neural networks can be realised in artificial networks through simple learning rules. Secondly, this research gives us an indication on how the brain has implemented certain tasks. Direct use of $\mathrm{tl}$ is information, combined with learning rules, may be a more successful way to design neural networks, than using learning alone.

In computer science, neural networks are regarded as (non)deterministic cellular automata, able to perform any task we wish to give it, using explicit parallelism. Important questions here are what the optimal network architecture is for a given task, as well as how different learning rules affect the performance of a network.

Finally there is theoretical physics, which views neural networks as interacting nonlinear dynamical systems. Using techniques from (nonequilibrium) statistical mechanics, research in this direction has revealed the stable states of a system, the domains of attraction for retrieval, the impact of noise on the system, phase transitions and chaotic behaviour.

In The Netherlands, some of the above mentioned fields have a long tradition. In particular there is a long tradition in research on neural information processing in sensory and motor systems in biological systems. The techniques used include both theoretical tools (e.g. systems theory, statistical physics) as well as experimental techniques, such as psychophysics and electrophysiological techniques. The biological research was done both on lower animals, such as insects, as well as higher animals as cats and primates. Most research on neural information processing in The Netherlands has been coordinated by the Foundation of Biophysics. In this foundation scientists with a background in physics, mathematics and biology have been collaborating for almost 60 years on experimental research as well as on neural modelling. 


\section{The foundation of neural networks}

In January 1990, a new research program on neural networks was initiated. In this program, the research is strongly focused towards those areas in which Holland has a tradition, i.e., in sensory and motor systems. Most neural network research in The Netherlands collaborates through this program. It is funded by the Dutch Ministry of Economics, by Dutch Universities and by industry and operates on an annual budget of Mfl. 2.5.

The coordination of this research program is in the hands of the two authors, who act as program manager and director, respectively, of this research program. Advise is given by the Industrial Advisory Committee (IAC) and Research Council. Each industrial partner has a delegate in the IAC. The main function of the IAC is to advise about possible applications in neural networks. The main task of the Research Council is to give an independent backing of the scientific quality of the research projects that are proposed by the research coordinators at the various universities. The members of the Research Council are invited among the top-leaders in the field in the USA and Europe.

Presently, the universities participating in the project are the Universities of Leiden (cognition), Amsterdam (robotics), Utrecht (3-D vision and audition) and Nijmegen (audition, sensor fusion, motor control, and computer science) which collaborate through a number of well-connected projects. Other universities may join in the future. The overall coordination is done by a new foundation, called the Foundation for Neural Networks (SNN). The SNN supports three main lines of research which are to a large extent complementary. The objectives cover part of the fundamental aspects of information processing in biological and artificial systems and aim at applications in particular areas of artificial intelligence.

The industrial partners that are participating in this research project have a very different background. Their interest varies from software applications (BSO, VOLMAC, and Digital Equip- ment Coorporation) to hardware applications and VLSI implementation of neural networks (ARCOBEL/PARSYTEC, Hewlett \& Packard, Philips (Paris)). Other industrial partners are more interested in new algorithms and theories of neural networks for their own applications (e.g. Shell, Digital Equipment Coorporation). Looking forward to 1992, when the European market will be open, we already are seeking contacts and collaboration with interested industries and similar research groups in Europe.

\section{SNN research activies}

\subsection{Storage and retrieval of information with neural networks}

Storage and retrieval of information with neural networks and the efficiency of information processing in distributed parallel networks, depends on several aspects such as the learning rule, the number of hierarchical layers in the network, and the number of elements in each network layer. In order to increase the storage capacity and the performance of neural networks different learning rules and related architectures will be developed and tested. The structure and architecture of the neural network are hard to separate from the learning rule since the former are partly the result of the latter. Research on these issues will be done at the University of Leiden.

Different models on neural networks and their implementation in software will be evaluated from the point of view of current computational models in computer science. At the Department of Computer Science at the University of Nijmegen the expressive power of different types of neural network models that are currently in use (e.g. Hopfield model, Boltzmann machine, (multi-layer) perceptron), the possibility of reasoning about the model and the relationship of the model with respect to other computational models in computer science will be investigated theoretically. The aim of this project is to gain insight in the properties of dif- 
ferent network types such that it will be possible to give a specific network architecture within the specifications of a particular problem.

\subsection{Sensory information processing}

An important aspect of this research line will be the development of networks that are able to perform invariant pattern recognition. The field of applicability here is audition as well as vision. For vision, all networks available now are very well able to store and retrieve patterns that are presented in a particular orientation and at a particular position in space. The recognition of objects independent of their size, orientation, and viewing angle is one of the goals.

For audition neural networks will be developed that perform part of the low-level auditory information processing, similar to the artificial cochlea developed by Carver Mead. Some of the applications are directed to improve the usefulness of artificial electrical stimulation in the cochlea with multi-electrodes in patients with a pathology in the peripheral auditory pathway. Moreover, the problem of speech recognition will be addressed especially with regard to the problem of speaker dependence.

The perception and storage of 3-D images is another field of attention. Central issues here are the modelling of feature detectors in the visual cortex (geometry engines), storage possibilities of a 3D image as stereo 2-D and viewpoint invariant characterisation of objects. This last subject is also very relevant in the study of optic flow where the separation of ego-motion from invariant characteristics of objects in the visual field is the central theme. Contrary to most neural network models that use general neuron models (e.g. of the McCulloch-Pitts type), the neurons in this projects will be specialised in the sense that they perform local derivatives on the optic flow (up to fourth order). The activity in the neural network therefore provides a jet-expansion in Taylor coefficients that allow a reconstruction of, for example, solid shape. This information can also be used to discriminate between object-motion and selfmotion which is important for the development of neural networks for navigation of autonomous systems in a cluttered environment.

Usually biological and artificial systems use multiple sources of sensory information. The reason is that any sensory system probes only a limited part of the environment. However, together the different sensory modalities provide a rather complementary "view" of the environment. In this line of research we will investigate how the fusion of multi-sensory information can be acquired in a distributed parallel system by selforganizing processes. Sensor fusion may be used for the control of autonomous systems but is equally important for other applications, e.g. for expert systems, where decisions have to be made using fuzzy or incomplete information.

\subsection{Coordination of movements}

Issues for research on the coordination of redundant manipulators are:

- the coordination of a (robot) manipulator based on an "internal representation" of the environment which is constructed with sensory information and which serves as a database specifying the position and velocity of objects in the environment,

- studies on control of redundant manipulators and inverse dynamics in biological systems, - self-organizing process for coordination and calibration of movements.

\section{Other neural network research}

There are various small research activities on neural networks in The Netherlands which are not united in SNN. Three of these should be mentioned:

- So far, there has not been much activity with respect to VLSI implementation of neural networks in The Netherlands. Only very recently a start has been made at the Universities in Eind- 
hoven, Delft and Twente. These activities will be part of the European JESSI program that is scheduled to start in summer 1990.

- In the field of neural networks for combinatorial optimization, we would like to mention the original work of Aarts and Korst at the Philips Research Laboratories on Boltzmann machines.

- At the University of Groningen, there is a long tradition of research on the visual system of the fly. Neural network research here uses adaptive visual strategies and recurrent pattern recognition techniques for the construction of artificial visual systems. This group also plays a leading role in the analysis of cochlear models that take a proper account of nonlinear and active behavior of the sensor. Research on the design of an artificial cochlea and of a visual speed detection system within SNN is presently being discussed.

\section{Conclusion}

This brief overview of research activities in neurocomputing in The Netherlands has mainly focused on artificial neural networks. However, considerable effort on biological neural networks is done at several universities. This interdisciplinary collaboration with biologists, physicists and mathematicians, which has been developed within the Foundation for Biophysics, will continue to provide an active collaboration in the future.

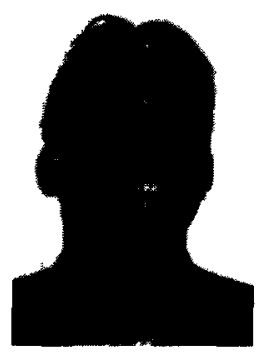

Dr. H.J. Kappen studied High Energy Physics at the University of Groningen, The Netherlands, and completed his Ph.D. in the same field in 1987 at the Rockefeller University in New York. From 1987 until 1989 he worked at the Philips Research Laboratories in Eindhoven, The Netherlands. Presently, he is working at the Laboratory of Medical Physics and Biophysics of the University of Nijmegen, The Netherlands, as a program manager of the Foundation for Neural Networks. His duties include management of the research activities in this Foundation as well as active research in the field of neural networks.

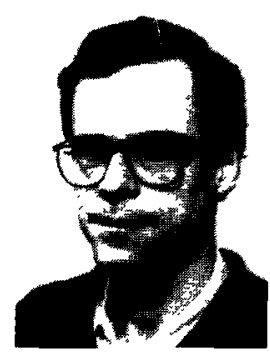

Professor Dr. C. Gielen is a professor in Biophysics at the University of Nijmegen since 1988. After obtaining his Masters Degree in experimental physics at the University of Nijmegen in 1976, he obtained his Ph.D. in 1980 with a research project on visual information processing in primates. After his Ph.D. he worked at the University of Utrecht on the general theme of information processing in biological organisms with special emphasis on the sensory coordination of movements. In the period from 1980 to 1988 he spent several years at Northwestern University (Chicago/Evanston) and at the University of California at Los Angeles (UCLA). 